\title{
Pre-mortem Diagnosis of Sporadic Creutzfeldt-Jakob Disease in Practice
}

\author{
Elina Ziukelis ${ }^{1}$ and James Gome ${ }^{2}$ \\ ${ }^{1}$ St Vincent's Health Australia Ltd Fitzroy \\ ${ }^{2}$ Deakin University
}

January 10, 2021

\begin{abstract}
We describe a case of Sporadic Creutzfeldt-Jakob disease (sCJD) and discuss our evidence-based diagnostic process.

Hosted file

Manuscript.pdf available at https://authorea.com/users/388447/articles/503251-pre-mortemdiagnosis-of-sporadic-creutzfeldt-jakob-disease-in-practice
\end{abstract}




\section{Definite Sporadic Creutzfeldt-Jakob Disease}

Diagnosed by standard neuropathological techniques; and/or immunocytochemically; and/or Western blot confirmed protease-resistant PrP; and /or presence of scrapie-associated fibrils.

Probable Sporadic Creutzfeldt-Jakob Disease

Neuropsychiatric disorder plus positive RT-QulC in cerebrospinal fluid (CSF) or other tissues

$$
\underline{\mathrm{OR}}
$$

Rapidly progressive dementia; and at least two out of the following four clinical features:

1. Myoclonus

2. Visual or cerebellar signs

3. Pyramidal/extrapyramidal signs

4. Akinetic mutism

AND a positive result on at least one of the following laboratory tests

- a typical EEG (periodic sharp wave complexes) during an illness of any duration

- a positive 14-3-3 CSF assay in patients with a disease duration of less than 2 years

- High signal in caudate/putamen on magnetic resonance imaging (MRI) brain scan or at least two cortical regions (temporal, parietal, occipital) either on diffusion-weighted imaging (DWI) or fluid attenuated inversion recovery (FLAIR)

AND without routine investigations indicating an alternative diagnosis.

Possible Creutzfeldt-Jakob Disease

Progressive dementia; and at least two out of the following four clinical features:

1. Myoclonus

2. Visual or cerebellar signs
3. Pyramidal/extrapyramidal signs

4. Akinetic mutism

AND absence of positive result for any of the 4 tests above that would classify a case as

"probable"

AND duration of illness less than two years

AND without routine investigations indicating an alternative diagnosis. 


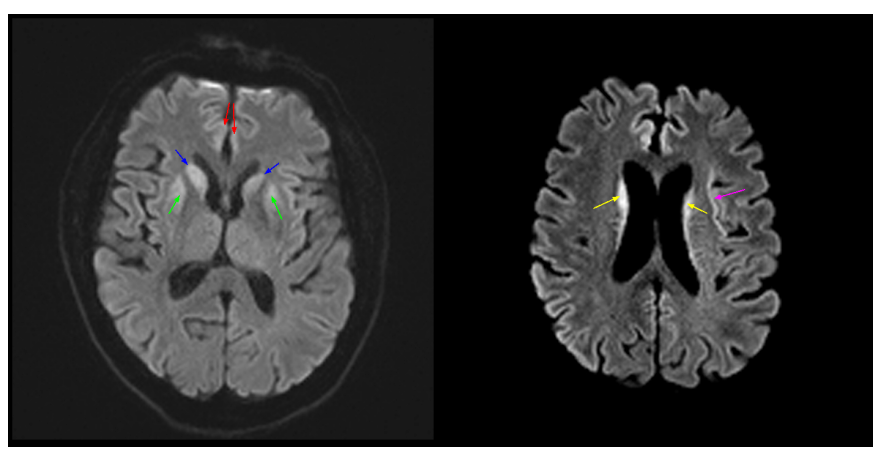

